\title{
PID controller design for fractional-order systems with time delays
}

\author{
Hitay Özbay ${ }^{\mathrm{a}, *}$, Catherine Bonnet ${ }^{\mathrm{b}}$, André Ricardo Fioravanti ${ }^{\mathrm{b}}$ \\ ${ }^{a}$ Dept. of Electrical and Electronics Eng., Bilkent University, Bilkent 06800 Ankara, Turkey \\ ${ }^{\mathrm{b}}$ INRIA Saclay - Île-de-France, Domaine de Voluceau, B.P. 105, 78153 Le Chesnay Cedex, France
}

\section{A R T I C L E I N F O}

\section{Article history:}

Received 28 November 2008

Received in revised form

19 September 2011

Accepted 19 September 2011

Available online 22 November 2011

\begin{abstract}
A B S T R A C T
Classical proper PID controllers are designed for linear time invariant plants whose transfer functions are rational functions of $s^{\alpha}$, where $0<\alpha<1$, and $s$ is the Laplace transform variable. Effect of input-output time delay on the range of allowable controller parameters is investigated. The allowable PID controller parameters are determined from a small gain type of argument used earlier for finite dimensional plants.
\end{abstract}

(c) 2011 Elsevier B.V. All rights reserved.

\section{Keywords:}

Fractional order systems

Time delay

PID controller

Stability analysis

Controller synthesis

\section{Introduction}

Fractional order system models have been widely studied over the past two decades (see e.g., [1-7] and their references), where stability analysis and controller design problems are studied. Another line of research in this context is the design of fractional order controllers, including fractional order PID controllers, for fractional order as well as rational (finite dimensional) systems [8-14].

Fractional order systems appear in various engineering applications, see, e.g., [15-20]. It is interesting to see that they might appear in two ways. First, through theoretical modeling of physical phenomena and second from frequency domain experiments when traditional integer order models do not fit the data (for instance when Bode diagrams do not show slopes of integer multiples of $20 \mathrm{~dB} /$ decade [21]). Many fields are concerned. In electricity, models of polarization emittance of metal electrodes [22] as well as capacitor models (based on purely empirical Curie's law of 1889) [23] are of fractional type. In material sciences, fractional order derivatives are used to model visco-elastic materials [24], non-laminated ferromagnetic components [25] or magnetic core coils [21]. Other physical phenomena such as heat conduction [26] or flexible structures [27] give rise to transfer functions with fractional powers of $s$ (typically square root of $s$ ).

\footnotetext{
* Corresponding author. Tel.: +90 312290 1449; fax: +90 3122664192.

E-mail addresses: hitay@bilkent.edu.tr (H. Özbay), Catherine.Bonnet@inria.fr (C. Bonnet), Andre.Fioravanti@inria.fr (A.R. Fioravanti).
}

The topic of the present work is the design of classical proper PID controllers for fractional order systems.

Many different PID controller design techniques are available for rational (finite dimensional) systems with time delays; e.g. [28-31]. In this paper, we extend the approach of [28] to fractional order systems with time delays.

The class of plants considered and the feedback control problem studied are defined in Section 2. The proposed PID controller design method is described in Section 3. A numerical example is given in Section 4, and concluding remarks are made in Section 5.

\section{Problem definition}

Consider the standard single input-single output feedback system shown in Fig. 1, where $C$ is the controller to be designed for the plant $P$.

We assume that the plant is linear and time invariant. Its dynamical behavior is represented by the transfer function

$P(s)=\mathrm{e}^{-h s} \frac{G\left(s^{\alpha}\right)}{s^{\alpha}-p}$

where $s$ is the Laplace transform variable, $h>0$ is the total input-output time delay, $\alpha \in(0,1)$ is the fractional order, $p \geq 0$ ( $p^{1 / \alpha}$ being the location of the unstable pole of the plant), and $G(w)$ is a rational stable transfer function in the variable $w=s^{\alpha}$ with $G(p) \neq 0$ and $G(0) \neq 0$. Such a plant was considered with $h=0$ in [25] when modeling non-laminated electromagnetic suspensions. 


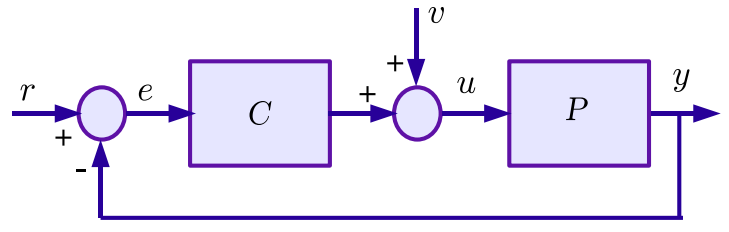

Fig. 1. Standard feedback system.

It is clear that we need $G(0) \neq 0$ for stabilizability of $(1)$ by a controller which includes an integrator. We assume that $\alpha$ is a rational number, i.e., we are restricting ourselves to the class of fractional systems of commensurate order, [6]. There is a simple stability test for this type of systems, which can be seen below.

Given all the parameters of plant (1), our goal is to design a classical Proportional + Integral + Differential (PID) controller in the form

$C(s)=K_{p}+\frac{K_{i}}{s}+K_{d} \frac{s}{\tau_{d} s+1}$

where $K_{p}, K_{i}, K_{d}$ are free parameters and $\tau_{d}$ is an arbitrarily small positive number making the controller proper.

The feedback system formed by the controller $C$ and the plant $P$ is stable if $(1+P C)^{-1}, C(1+P C)^{-1}$ and $P(1+P C)^{-1}$ are stable transfer functions. These transfer functions are indeed fractional delay systems of retarded type and it has been proven [32] that $\mathrm{H}_{\infty}$-stability of these systems is equivalent to their BIBO-stability, a necessary and sufficient condition being that the system has no poles in the right half-plane (including no pole of fractional order at $s=0$ ) and a numerical algorithm to test this property is available in [33]. In the case of fractional systems of commensurate order, checking stability can be done as follows (see e.g. $[3,6]$ ). Let $w=s^{\alpha}$ and assume that $T(w)$ is a rational function with poles $w_{1}, \ldots, w_{n}$. Enumerate the poles so that $w_{1}, \ldots, w_{2 n_{c}}$ are complex conjugate, with $w_{n_{c}+k}=\bar{w}_{k}$ and $w_{k}=\left|w_{k}\right| \mathrm{e}^{\mathrm{j} \theta_{k}}$ where $\theta \in(0, \pi)$ for $k=1, \ldots, n_{c}$, and $w_{2 n_{c}+1}, \ldots, w_{n}$ are real. Then, the system $T\left(s^{\alpha}\right)$ is stable if and only if

$\alpha \frac{\pi}{2}<\theta_{k}$ for $k=1, \ldots, n_{c}$, and

$w_{k}<0$ for $k=2 n_{c}+1, \ldots, n$.

We say that $C$ is a stabilizing controller for the plant $P$ if the feedback system formed by this pair is stable.

\section{PID controller design}

In this section, we design classical PID controllers in form (2) for plant (1). As in [28], the design will be done in two steps: first, PD controllers will be investigated, and then the integral action will be added.

\subsection{PD controller design}

A typical PD controller can be written in the form

$C_{p d}(s)=K_{p}\left(1+\widetilde{K}_{d} \frac{s}{\tau_{d} s+1}\right)$.

We can express the non-delayed part of the plant as the ratio of two stable factors:

$P(s)=\mathrm{e}^{-h s} Y(s)^{-1} X(s)$ with $Y(s):=\frac{s^{\alpha}-p}{s^{\alpha}+x}$

$X(s):=\frac{G\left(s^{\alpha}\right)}{s^{\alpha}+x}$ where $x>0$ is the free parameter. While it is an arbitrary positive number at this stage, $x$ plays an important role in the controller design.

With the notation introduced in (4), the feedback system stability is equivalent to stability of $U^{-1}$, where

$U(s):=Y(s)+\mathrm{e}^{-h s} X(s) C_{p d}(s)$.

Inserting $C_{p d}, X$ and $Y$ into (5), we have

$U(s)=1-\frac{(p+x)}{s^{\alpha}+x}+\mathrm{e}^{-h s} \frac{G\left(s^{\alpha}\right)}{s^{\alpha}+x} K_{p}\left(1+\widetilde{K}_{d} \frac{s}{\tau_{d} s+1}\right)$.

By choosing

$K_{p}=(p+x) G(0)^{-1}$

we obtain

$$
\begin{aligned}
U(s)= & 1-\frac{(p+x)}{s^{\alpha}+x} \\
& \times\left(1-\mathrm{e}^{-h s} G\left(s^{\alpha}\right) G(0)^{-1}\left(1+\widetilde{K}_{d} \frac{s}{\tau_{d} s+1}\right)\right) \\
= & 1-\frac{(p+x) s^{\alpha}}{s^{\alpha}+x} \\
& \times\left(\frac{1-\mathrm{e}^{-h s} G\left(s^{\alpha}\right) G(0)^{-1}}{s^{\alpha}}-\frac{\widetilde{K}_{d} \mathrm{e}^{-h s} G\left(s^{\alpha}\right) s^{1-\alpha}}{G(0)\left(\tau_{d} s+1\right)}\right) .
\end{aligned}
$$

Since $\left\|\frac{s^{\alpha}}{s^{\alpha}+x}\right\|_{\infty}=1$ for all $x>0$, by the small gain theorem, $U^{-1}$ is stable if

$$
\begin{aligned}
& \left\|\left(\frac{1-\mathrm{e}^{-h s} G\left(s^{\alpha}\right) G(0)^{-1}}{s^{\alpha}}\right)-\widetilde{K}_{d} \mathrm{e}^{-h s} G\left(s^{\alpha}\right) G(0)^{-1} \frac{s^{1-\alpha}}{\tau_{d} s+1}\right\|_{\infty} \\
& \quad<\frac{1}{(p+x)} .
\end{aligned}
$$

The following results are immediate consequences of the above discussion.

Lemma 1. For plant (1) there exists a stabilizing proportional controller, $C(s)=K_{p}$, if

$p<\left\|\frac{1-\mathrm{e}^{-h s} G\left(s^{\alpha}\right) G(0)^{-1}}{s^{\alpha}}\right\|_{\infty}^{-1}=: \psi_{0}$.

When (8) holds, all proportional controllers in the form (6) are stabilizing, where $x$ satisfies $0<x<\left(\psi_{0}-p\right)$.

Lemma 2. Suppose there exist $\widetilde{K}_{d} \in \mathbb{R}$ and $\tau_{d}>0$, such that

$$
\begin{aligned}
p< & \|\left(\frac{1-\mathrm{e}^{-h s} G\left(s^{\alpha}\right) G(0)^{-1}}{s^{\alpha}}\right)-\widetilde{K}_{d} \mathrm{e}^{-h s} G\left(s^{\alpha}\right) G(0)^{-1} \\
& \times \frac{s^{1-\alpha}}{\tau_{d} s+1} \|_{\infty}^{-1}=: \psi_{d} .
\end{aligned}
$$

Then, the controller $C_{p d}(s)=K_{p}\left(1+\widetilde{K}_{d} \frac{s}{\tau_{d} s+1}\right)$ is a stabilizing controller for plant (1) with $K_{p}=(p+x) G(0)^{-1}$ for all $x$ satisfying $0<x<\left(\psi_{d}-p\right)$.

From the PD controller design method proposed in Lemma 2, we see that the allowable values of the proportional gain are in the range

$K_{p}^{\min }:=p G(0)^{-1}<K_{p}<\psi_{d} G(0)^{-1}=: K_{p}^{\max }$. 
Therefore, we would like to maximize $\psi_{d}$ in order to maximize the allowable range for $K_{p}$. This problem is equivalent to finding the optimal $\widetilde{K}_{d} \in \mathbb{R}$ so that

$$
\begin{aligned}
\psi_{d}^{-1}= & \|\left(\frac{1-\mathrm{e}^{-h s} G\left(s^{\alpha}\right) G(0)^{-1}}{s^{\alpha}}\right)-\widetilde{K}_{d} \mathrm{e}^{-h s} G\left(s^{\alpha}\right) G(0)^{-1} \\
& \times \frac{s^{1-\alpha}}{\tau_{d} s+1} \|_{\infty}
\end{aligned}
$$

is minimized for a given fixed $\tau_{d}>0$. A similar problem has been studied in [30] for the case $\alpha=1$, i.e., for rational systems with time delays. In general, minimization of $\psi_{d}^{-1}$ is a two-dimensional search: for each fixed $\widetilde{K}_{d} \in \mathbb{R}$, compute the infinity norm by a frequency sweep. In [30], it is shown that, for a large class of rational systems with time delays, this computation can be reduced to a one dimensional search. Currently, we do not know if a similar result can be obtained for the class of plants studied here; we leave this problem open for a future study.

Once $\psi_{d}$ is maximized, we would like to choose $K_{p}$ so that the gain margin is maximized, i.e.,

$\min \left\{\frac{K_{p}}{K_{p}^{\min }}, \frac{K_{p}^{\max }}{K_{p}}\right\}$

is maximized, [34]. Clearly, the optimal choice is $K_{p}^{\mathrm{opt}}=$ $\sqrt{K_{p}^{\min } K_{p}^{\max }}$, i.e.

$K_{p}^{\mathrm{opt}}=\sqrt{p \psi_{d}} G(0)^{-1}$.

\subsection{Adding integral action to the PD controller}

Assume that condition (9) of Lemma 2 is satisfied and hence a stabilizing PD controller $C_{p d}$ can be found for plant (1). We now try to find

$C_{i}(s)=\frac{K_{i}}{s}$

so that $C_{p i d}(s)=C_{p d}(s)+C_{i}(s)$ is a stabilizing controller for the plant. This is a two step design process and it works as follows; see e.g. $[28,35]$. Define

$H(s):=P(s)\left(1+P(s) C_{p d}(s)\right)^{-1}$

and note that $H(0)=G(0) / x$ which is non-zero by the assumption that $G(0) \neq 0$ and by design $x>0$. If $C_{i}$ defined by (12) is a stabilizing controller for the "new plant" $H(13)$, then $C_{p i d}$ is a stabilizing controller for the original plant $P$. Now let

$K_{i}:=\gamma H(0)^{-1}, \quad$ with $\gamma>0$

then

$$
\begin{aligned}
(1 & \left.+C_{i}(s) H(s)\right)^{-1} \\
& =\frac{s}{s+\gamma}\left(1+\frac{\gamma s^{\alpha}}{s+\gamma}\left(\frac{H(s) H(0)^{-1}-1}{s^{\alpha}}\right)\right)^{-1} .
\end{aligned}
$$

Let us define

$R_{\alpha}(\gamma):=\left\|\frac{\gamma s^{\alpha}}{s+\gamma}\right\|_{\infty}$

Then by the small gain theorem $C_{i}(s)=\gamma H(0)^{-1} / s$ is a stabilizing controller for $H(s)$ if

$0<R_{\alpha}(\gamma)<\left\|\frac{H(s) H(0)^{-1}-1}{s^{\alpha}}\right\|_{\infty}^{-1}$.

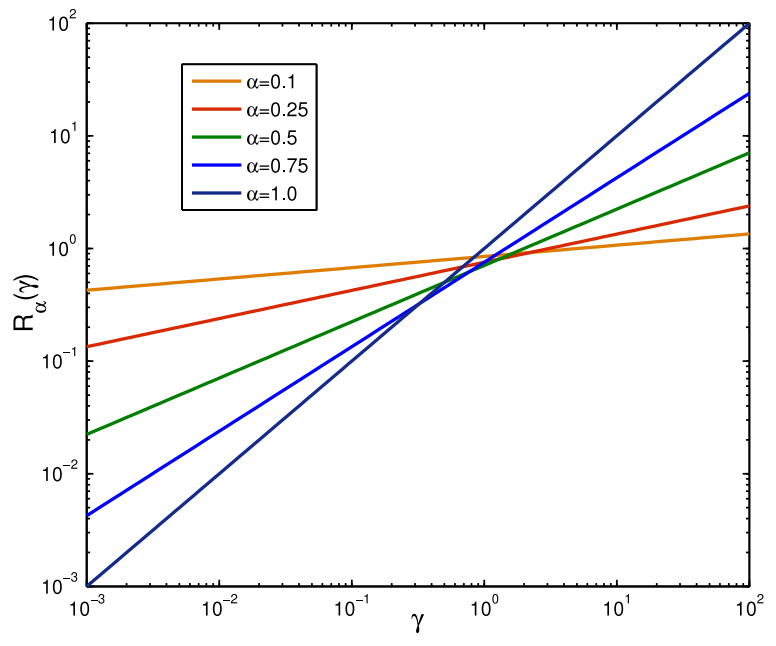

Fig. 2. $R_{\alpha}(\gamma)$ versus $\gamma$.

Note that for $\alpha=1$ we have $R_{1}(\gamma)=\gamma$, and for the rational case the function $\left(H(s) H(0)^{-1}-1\right) / s$ is stable. However, when $H(s)$ is a fractional transfer function, $\left(H(s) H(0)^{-1}-1\right) / s$ might be unstable due to problems of boundedness at zero. Therefore, writing

$$
\begin{aligned}
\left(1+C_{i}(s) H(s)\right)^{-1} & \\
= & \frac{s}{s+\gamma}\left(1+\frac{\gamma s}{s+\gamma}\left(\frac{H(s) H(0)^{-1}-1}{s}\right)\right)^{-1},
\end{aligned}
$$

rather than (15), and then applying the small gain theorem, as was done in [28], does not work in the case of fractional systems. So, we have to compute $R_{\alpha}(\gamma)$ as a function of $\gamma$ for the specific $\alpha$ value appearing in the plant transfer function. It is a simple exercise to show that

$R_{\alpha}(\gamma)=\alpha^{\alpha / 2}(1-\alpha)^{(1-\alpha) / 2} \gamma^{\alpha}$.

The graphs of $R_{\alpha}(\gamma)$ versus $\gamma$ for different values of $\alpha$ are shown in Fig. 2. Another observation we can make from (15) is that if $\left\|H(s) H(0)^{-1}-1\right\|_{\infty}<1$ then all $C_{i}(s)=\gamma H(0)^{-1} / s$ stabilize $H$, for any $\gamma>0$.

The above discussion is summarized with the following results.

Lemma 3. Assume that condition (8) of Lemma 1 is satisfied and the proportional controller $K_{p}=(p+x) G(0)^{-1}$ is designed to stabilize the plant $P(s)=\mathrm{e}^{-h s} G\left(s^{\alpha}\right)\left(s^{\alpha}-p\right)^{-1}$. Then the PI controller

$C_{p i}(s)=K_{p}+\frac{\gamma H(0)^{-1}}{s}=\left((p+x)+\frac{\gamma x}{s}\right) G(0)^{-1}$

is a stabilizing controller for the plant $P$ for all $\gamma$ satisfying

$0<R_{\alpha}(\gamma)<\left\|\frac{H(s) H(0)^{-1}-1}{s^{\alpha}}\right\|_{\infty}^{-1}$

where $H(s)=P(s)\left(1+K_{p} P(s)\right)^{-1}$.

Lemma 4. Assume that condition (9) is satisfied for some $\widetilde{K}_{d} \in \mathbb{R}$ and $\tau_{d}>0$. Let $C_{p d}$ be a stabilizing controller for the plant, $P(s)=$ $\mathrm{e}^{-h s} G\left(s^{\alpha}\right)\left(s^{\alpha}-p\right)^{-1}$, as designed in Lemma 2. Then the PID controller

$$
\begin{aligned}
C_{p i d}(s) & =C_{p d}(s)+\frac{\gamma H(0)^{-1}}{s} \\
& =\left((p+x)\left(1+\widetilde{K}_{d} \frac{s}{\tau_{d} s+1}\right)+\frac{\gamma x}{s}\right) G(0)^{-1}
\end{aligned}
$$


is a stabilizing controller for $P$ for all $\gamma$ satisfying

$0<R_{\alpha}(\gamma)<\left\|\frac{H(s) H(0)^{-1}-1}{s^{\alpha}}\right\|_{\infty}^{-1}$

where $H(s)=P(s)\left(1+C_{p d}(s) P(s)\right)^{-1}$.

The above PI and PID controller design methods lead to an interesting question: what are the optimal choices of $x>0$ such that the ranges of allowable $\gamma$, i.e. the right hand sides of (21) and (23), are the largest possible? For example, in the PI design, for each fixed $x$ in the range $0<x<\left(\psi_{0}-p\right)$, one can compute the upper bound in (21) numerically. Therefore, the largest allowable $\gamma$ range and the corresponding optimal $x$ can be found from a one dimensional numerical search. Clearly, it is not possible to find an analytical solution for this problem.

On the other hand, we can find a suboptimal analytical solution as follows. Recall that $H(0)=G(0) / x$ and

$H(s)=\mathrm{e}^{-h s} G\left(s^{\alpha}\right)\left(\left(s^{\alpha}-p\right)+x-x+(p+x) G(0)^{-1} \mathrm{e}^{-h s} G\left(s^{\alpha}\right)\right)^{-1}$.

Then we have

$$
\begin{aligned}
& \frac{H(s) H(0)^{-1}-1}{s^{\alpha}} \\
& =\frac{\frac{\mathrm{e}^{-h s} G\left(s^{\alpha}\right) G(0)^{-1} x}{s^{\alpha}+x}\left(1-\left(\frac{(p+x) s^{\alpha}}{s^{\alpha}+x}\right) \frac{1-\mathrm{e}^{-h s} G\left(s^{\alpha}\right) G(0)^{-1}}{s^{\alpha}}\right)^{-1}-1}{s^{\alpha}} \\
& =\frac{\frac{p}{\left(s^{\alpha}+x\right)}\left(\frac{1-\mathrm{e}^{-h s} G\left(s^{\alpha}\right) G(0)^{-1}}{s^{\alpha}}\right)-\frac{1}{s^{\alpha}+x}}{1-\frac{(p+x) s^{\alpha}}{s^{\alpha}+x}\left(\frac{1-\mathrm{e}^{-h s} G\left(s^{\alpha}\right) G(0)^{-1}}{s^{\alpha}}\right)} .
\end{aligned}
$$

Recall that

$\psi_{o}=\left\|\frac{1-\mathrm{e}^{-h s} G\left(s^{\alpha}\right) G(0)^{-1}}{s^{\alpha}}\right\|_{\infty}^{-1}$.

So, from the above

$\left\|\frac{H(s) H(0)^{-1}-1}{s^{\alpha}}\right\|_{\infty} \leq \frac{p \psi_{o}^{-1}+1}{x}\left(1-(p+x) \psi_{o}^{-1}\right)^{-1}$.

Thus we have the following lower bound for the upper bound in (21),

$\tilde{\gamma}:=x \frac{\psi_{o}-(p+x)}{\psi_{0}+p} \leq\left\|\frac{H(s) H(0)^{-1}-1}{s^{\alpha}}\right\|_{\infty}^{-1}$.

Now we can maximize $\tilde{\gamma}$ by an appropriate choice of $x$. It is a simple exercise to show that the optimal choice of $x$ maximizing $\tilde{\gamma}$ is

$x_{\mathrm{opt}}=\frac{\psi_{o}-p}{2}$

and the corresponding maximal $\tilde{\gamma}$ is $\frac{x_{\mathrm{opt}}^{2}}{\psi_{0}+p}$. This means that, by (19), $\gamma$ should be in the range

$0<\gamma<\frac{c_{\alpha} x_{\mathrm{opt}}^{2 / \alpha}}{\left(\psi_{0}+p\right)^{1 / \alpha}}=: \gamma_{\max }$

where $c_{\alpha}:=\left(\sqrt{\alpha}(1-\alpha)^{(1-\alpha) / 2 \alpha}\right)^{-1}$.

For example $c_{0.5}=2$. We propose to choose

$\gamma_{\mathrm{opt}}:=\frac{\gamma_{\mathrm{max}}}{2}$

as the (sub)optimal $\gamma$ value to be used in the PI controller. Inserting (25) into the PI controller expression (20), we obtain

$C_{p i}(s)=\left(1+\frac{\gamma_{\mathrm{opt}}}{s}\right) x_{\mathrm{opt}} G(0)^{-1}$

as the suboptimal PI controller, where $x_{\mathrm{opt}}$ is given by (25) and $\gamma_{\mathrm{opt}}$ is determined from (26) to (27).

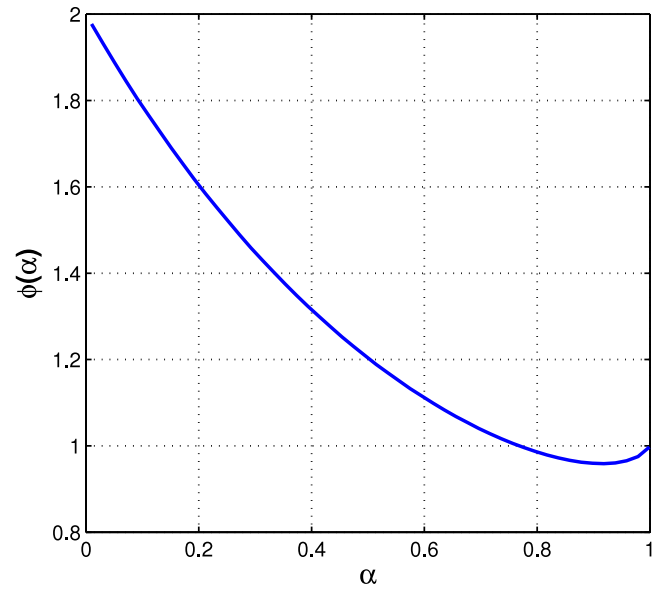

Fig. 3. $\phi(\alpha)=h^{-\alpha} \psi_{0}^{-1}$ versus $\alpha$.

\section{Examples}

Example 1. We will first consider the plant

$P(s)=\frac{\mathrm{e}^{-h s}}{s^{\alpha}-p}, \quad$ with $h>0, p \geq 0$

and design PID controllers using the method developed in Section 3.

For P and PI controller design we need to compute the quantity

$\psi_{o}=\left\|\frac{1-\mathrm{e}^{-h s} G\left(s^{\alpha}\right) G(0)^{-1}}{s^{\alpha}}\right\|_{\infty}^{-1}$.

When $\alpha=1$, we have $\psi_{0}=h^{-1}$. In the case $0<\alpha<1$, we compute $\psi_{o}$ from

$\psi_{o}^{-1}=\sup _{\omega \in \mathbb{R}} \frac{\left|1-\mathrm{e}^{-j h \omega}\right|}{\left|(j \omega)^{\alpha}\right|}=h^{\alpha} \sqrt{2} \sup _{\tilde{\omega} \in \mathbb{R}} \frac{\sqrt{1-\cos (\tilde{\omega})}}{\tilde{\omega}^{\alpha}}$.

Therefore,

$h^{-\alpha} \psi_{o}^{-1}=\sqrt{2} \sup _{\tilde{\omega} \in \mathbb{R}} \frac{\sqrt{1-\cos (\tilde{\omega})}}{\tilde{\omega}^{\alpha}}=: \phi(\alpha)$.

Fig. 3 shows how $\phi(\alpha)$ varies with $\alpha$. As expected, for $\alpha=1$ we have $\phi=1$. But it is interesting to observe that behavior of $\phi$ is not monotonic, and there is a minimum value near $\alpha=0.9$.

According to Lemma 1, there is a stabilizing controller for plant (29) if $p<\psi_{0}$, i.e., if

$p h^{\alpha}<\frac{1}{\phi(\alpha)}$

where $\phi(\alpha)$ is as shown in Fig. 3. In particular, for $\alpha=0.5$, we have $\phi=1.2$ and we can find a stabilizing proportional controller using Lemma 1 if

$h<\frac{1}{1.2^{2} p^{2}}=\frac{0.6944}{p^{2}}$.

Recall that the sufficient conditions of Section 3 are obtained using the small gain arguments, so there is some conservatism. We can also use the results of [36] and find that there exists a stabilizing proportional controller for all $h<h_{\max }$ as follows.

The stability for $h=0$ is guaranteed with $K_{p}>p$. When $h$ increases, the position of the infinite number of new poles poses no restriction, since for a delay system of retarded type (the closedloop $\left[P, K_{p}\right]$ is indeed a fractional delay system of retarded type) they appear in the left-half plane. The exact value of the delay for 
Table 1

\begin{tabular}{|c|c|c|c|c|c|c|c|c|c|c|}
\hline$\underset{\sim}{c}$ & 0.10 & 0.17 & 0.28 & 0.46 & 0.77 & 1.29 & 2.15 & 3.59 & 5.99 & 1.00 \\
\hline$\widetilde{h}_{\max }$ & 4.14 & 3.13 & 2.35 & 1.75 & 1.29 & 0.94 & 0.68 & 0.48 & 0.34 & 0.23 \\
\hline$h_{\max }[37]$ & 4.58 & 3.56 & 2.78 & 2.17 & 1.68 & 1.35 & 1.05 & 0.83 & 0.64 & 0.46 \\
\hline$K_{d}$ & 0.46 & 0.59 & 0.78 & 1.01 & 1.31 & 1.78 & 2.32 & 3.18 & 4.44 & 6.50 \\
\hline
\end{tabular}

which some poles cross the imaginary axis are related to the nonnegative real roots $\omega_{R}$ of the quasi-polynomial

$W(\omega)=\omega-p \sqrt{2 \omega}+p^{2}-K_{p}^{2}$

which leads to $\omega_{R}=K_{p}^{2}+p \sqrt{2 K_{p}^{2}-p^{2}}$.

The maximum delay $h$ is given by

$h=\frac{1}{\omega_{R}} \arcsin \left(\frac{\sqrt{2 \omega_{R}}}{2 K_{p}}\right)$

and, maximizing (31) with respect to $K_{p}>p$ results in $K_{p} \rightarrow p$, and hence $h \rightarrow h_{\max }$ with

$h_{\max }=\frac{\pi}{4 p^{2}} \approx \frac{0.7854}{p^{2}}$.

The value of $h_{\max }$ is exact, in the sense that if $h \geq h_{\max }$ then there does not exist a stabilizing proportional controller. Thus the level of conservatism in our approach is less than $12 \%$ (to be exact $(0.7854-0.6944) / 0.7854=0.1159)$.

The suboptimal PI controller $(28)$ for $P(s)=\frac{\mathrm{e}^{-h s}}{\sqrt{s}-p}$ can be computed from

$\psi_{o}=\frac{1}{1.2 \sqrt{h}}, \quad x_{\mathrm{opt}}=\frac{\psi_{o}-p}{2}$

$\gamma_{\mathrm{opt}}=\frac{1}{4}\left(\frac{\psi_{o}-p}{\psi_{0}+p}\right)^{2}\left(\frac{\psi_{o}-p}{2}\right)^{2}$.

In particular, when $p=0$, we have

$C_{p i}(s)=\frac{1}{2.4 \sqrt{h}}\left(1+\frac{1 / 16}{1.2^{2} h s}\right)$.

For the optimal PD controller proposed in Section 3, we need to find the optimal $\widetilde{K}_{d} \in \mathbb{R}$, say $\widetilde{K}_{d}^{\text {opt }}$, so that $\psi_{d}^{-1}$, (10), is minimized for a small fixed value of $\tau_{d}>0$.

Considering $h=1$, we calculated the optimal PD control which results in the parameters $\tau_{d}=4.2$ and $\widetilde{K}_{d}^{\text {opt }}=-1.7346$, and hence $\psi_{d}^{-1}=0.9873$. Then the optimal PD controller is given by

$G(0)^{-1} \sqrt{p \psi_{d}}\left(1+\widetilde{K}_{d}^{\mathrm{opt}} \frac{s}{\tau_{d} s+1}\right)$

where stability is assured for all systems with $p<\psi_{d}=1.0165$. Notice that with just the proportional controller, we could only guarantee stability for systems with $p<0.8333$, which indicates an increase of about $22 \%$.

Example 2. Now consider the following plant modeling a nonlaminated magnetic suspension system as studied in [25]:

$P_{2}(s)=\mathrm{e}^{-h s} \frac{G\left(s^{\alpha}\right)}{s^{\alpha}-p}=\mathrm{e}^{-h s} \frac{1}{\left(s^{\alpha}\right)^{5}+\left(s^{\alpha}\right)^{4}-c} \quad \alpha=0.5$

where $c$ is a positive real constant and in the ideal case $h=0$. This system has exactly one real positive pole and four poles in the lefthalf plane; see [25]. Hence, the techniques presented in Section 3 are applicable. We investigate the largest allowable time delay $h$ (which may exist due to communication constraints between the controller and the plant) for which the PD controller design technique proposed in this paper gives a stable feedback system.
Table 1 shows the results for 10 values of $c$ logarithmically spaced between 0.1 and 10 . For each one of those points, a PD controller that maximizes the allowable value of delay was calculated using the results of Section 3. The maximal allowable delay for which our technique finds an admissible PD controller is denoted by $\widetilde{h}_{\max }$. The optimal PD controller determined using the techniques of Section 3, has the proportional gain $K_{p}=c$, and $K_{d}$ is shown in Table 1 for various values of $c$, for the delay $\widetilde{h}_{\max }$. For the PD controllers designed, the exact value of maximal allowable delay, denoted by $h_{\max }$, can be calculated using the numerical techniques presented in [37]. We see that the degree of conservatism (i.e. the gap between $h_{\max }$ and $h_{\max }$ ) is low.

\section{Conclusions}

In this paper, we developed a method to design classical PID controllers (with proper derivative action) for a class of fractional order plants with time delays. The main idea behind this approach was to use the small gain type of arguments used in [28]. The fractional order plant is factored into a stable part and an unstable part, where the unstable part is in the form $\left(s^{\alpha}-p\right)^{-1}$ with $p>0$. There is no restriction on the stable part $G\left(s^{\alpha}\right)$ except that $G(0) \neq$ 0 and $G(p) \neq 0$. It may be possible to extend this method to fractional order plants with a higher degree unstable part, but in that situation there are some technical difficulties even for the case of rational plants; see [28] and its references.

The (sub)optimal PD and PI controller design method proposed here also works for rational plants with time delays and single pole in $\mathbb{R}_{+}$; see [30]. However, in the case of fractional systems, there is a major difference for the minimization of $\psi_{d}^{-1},(10)$ : when $\alpha \neq 1$ we cannot let $\tau_{d}=0$, because, otherwise $s^{1-\alpha}$ term multiplying $\widetilde{K}_{d}$ will make the norm equal to infinity unless $\widetilde{K}_{d}=0$. Therefore, the selection of a small positive $\tau_{d}$ plays an important role in this case, and hence, search for the optimal $\widetilde{K}_{d}$ and $\tau_{d}$ pair is more difficult compared to the problem studied in [30]. On the other hand, having a positive $\tau_{d}$ makes the "proper PD controller" a stable first order controller. So, in this sense optimization of $\widetilde{K}_{d}$ and $\tau_{d}$ solves the optimal first order stable controller design problem.

\section{Acknowledgment}

This work is supported in part by TUBITAK under grant no. $105 \mathrm{E} 156$ and by DPT-HAMiT project.

\section{References}

[1] H.-S. Ahn, Y.Q. Chen, I. Podlubny, Robust stability test of a class of linear timeinvariant interval fractional-order system using Lyapunov inequality, Applied Mathematics and Computation 187 (2007) 27-34.

[2] Y.Q. Chen, H.-S. Ahn, I. Podlubny, Robust stability check of fractional order linear time invariant systems with interval uncertainties, Signal Processing 86 (2006) 2611-2618.

[3] T.T. Hartley, C.F. Lorenzo, Dynamics and control of initialized fractional-order systems, Nonlinear Dynamics 29 (2002) 201-233.

[4] R. Malti, M. Aoun, O. Cois, A. Oustaloup, F. Levron, $H^{2}$ norm of fractional differential systems, in: Proceedings of ASME 2003 Design Engineering Technical Conferences and Computers and Information in Engineering Conference Chicago, IL, September 2003, DETC2003/VIB-48387. 
[5] D. Matignon, B. d'Andrea-Novel, Observer-based controllers for fractional differential systems, in: Proc. of the 36th IEEE Conference on Decision and Control, San Diego, CA, December 1997, pp. 4967-4972.

[6] D. Matignon, Stability properties for generalized fractional differential systems, ESAIM Proceedings Fractional Differential Systems Models Methods and Applications 5 (1998) 145-158.

[7] A.G. Radwan, A.M. Soliman, A.S. Elwakil, A. Sedeek, On the stability of linear systems with fractional-order elements, Chaos, Solitons \& Fractals (2007) doi:10.1016/j.chaos.2007.10.033.

[8] C. Bonnet, J.R. Partington, Stabilization of some fractional delay systems of neutral type, Automatica 43 (2007) 2047-2053.

[9] V. Feliu-Batlle, R. Rivas Perez, L. Sanchez Rodriguez, Fractional robust control of main irrigation canals with variable dynamic parameters, Control Engineering Practice 15 (2007) 673-686.

[10] P. Lanusse, H. Benlaoukli, D. Nelson-Gruel, A. Oustaloup, Fractional-order control and interval analysis of SISO systems with time-delayed state, IET Control Theory \& Applications 2 (2008) 16-23.

[11] M.P. Lazarevic, Finite time stability analysis of $P D^{a}$ fractional control of robotic time-delay systems, Mechanics Research Communications 33 (2006) 269-279.

[12] C. Ma, Y. Hori, Fractional-order control: theory and applications in motion control, IEEE Industrial Electronics Magazine (2007) 6-16. Winter.

[13] I. Podlubny, Fractional-order systems and $P^{\lambda} D^{\mu}$-controllers, IEEE Transactions on Automatic Contol 44 (1999) 208-214.

[14] D. Valerio, J.S. da Costa, Tuning of fractional PID controllers with Ziegler Nichols type rules, Signal Processing 86 (2006) 2771-2784.

[15] B.N. Narahari Achar, J.W. Hanneken, T. Clarke, Response characteristics of fractional oscillator, Physica A 309 (2002) 275-288.

[16] O.M.P. Agrawal, Solution for a fractional diffusion-wave equation defined in a bounded domain, Nonlinear Dynamics 29 (2002) 145-155.

[17] J.-L. Battaglia, O. Cois, L. Puisegur, A. Oustaloup, Solving an inverse heat conduction problem using a non-integer identified model, International Journal of Heat and Mass Transfer 44 (2001) 2671-2680.

[18] S.E. Hamamci, An algorithm for stabilization of fractional-order time delay systems using fractional-order PID controllers, IEEE Transactions on Automatic Control 52 (2007) 1964-1969.

[19] M.D. Ortigueira, Introduction to fractional linear systems, Part 1 : continuoustime case, IEE Proceedings-Vision, Image, Signal Processing 147 (2000) 62-70.

[20] M. Ostoja-Starzewski, H. Shahsavari, Response of a helix made of a fractional viscoelastic material, Journal of Applied Mechanics 75 (2008).
[21] I. Schäfer, K. Krüger, Modelling of coils using fractional derivatives, Journal of Magnetism and Magnetic Materials 307 (2006) 91-98.

[22] H. Sun, B. Onaral, A unified method to represent metal electrode polarization, IEEE Transactions on Biomedical Engineering BME30 (7) (1983) 399-406.

[23] S. Westerlund, Capacity theory, IEEE Transactions on Dielectrics and Electrical Insulation 1 (2) (1994) 826-839.

[24] S. Skaar, A. Michel, R. Miller, Stability of visco-elestic control systems, IEEE Transactions on Automatic Contol 33 (4) (1988) 348-357.

[25] C. Knospe, L. Zhu, Performance limitations of non-laminated magnetic suspension systems, IEEE Transactions on Control Systems Technology 19 (2) (2011) 327-336.

[26] JJ. Loiseau, H. Mounier, Stabilisation de l'quation de la chaleur commande en flux, ESIAM: Proceedings-Fractional Differential Systems: Models, Methods and Applications 5 (1998) 131-144.

[27] S. Manabe, A suggestion of fractional-order controller for flexible spacecraft attitude control, Nonlinear Dynamics 29 (2002) 251-268.

[28] A.N. Gündeş, H. Özbay, A.B. Özgüler, PID controller synthesis for a class of unstable MIMO plants with I/O delays, Automatica 43 (1) (2007) 135-142.

[29] C. Lin, O.-G. Wang, T.H. Lee, An improvement on multivariable PID controller design via iterative LMI approach, Automatica 40 (2004) 519-525.

[30] H. Özbay, A.N. Gündeş, Resilient PI and PD controller designs for a class of unstable plants with I/O delays, Applied and Computational Mathematics 6 (2007) 18-26.

[31] G.J. Silva, A. Datta, S.P. Bhattacharyya, PID Controllers for Time-Delay Systems, Birkhäuser, Boston, 2005.

[32] C. Bonnet, J.R. Partington, Analysis of fractional delay systems of retarded and neutral type, Automatica 38 (7) (2002) 1133-1138.

[33] C. Hwang, Y.-C Cheng, A numerical algorithm for stability testing of fractional delay systems, Automatica 42 (2006) 825-831.

[34] H. Özbay, Introduction to Feedback Control Theory, CRC Press LLC, Boca Raton FL, 2000.

[35] M. Vidyasagar, Control System Synthesis: A Factorization Approach, MIT Press, Cambridge MA, 1985.

[36] J.E. Marshall, H. Górecki, K. Walton, A. Korytowski, Time Delay Systems: Stability And Performance Criteria With Applications, Ellis Horwood, England, 1992.

[37] A.R. Fioravanti, C. Bonnet, H. Özbay, S.-I. Niculescu, Stability windows and unstable root-loci for linear fractional time-delay systems, The 18th IFAC World Congress, Milan, Italy, August 28-September 02, 2011, pp. $12532-12537$. 